\title{
DRONE INTEGRATED WEATHER SENSORS FOR AGRICULTURE PURPOSE
}

\author{
Dr. Anil Kumar D \\ Assistant Professor, Department of Electronics and Communication Engineering, \\ BMS Institute of Technology \& Management, Bengaluru, India

\section{Dr. Ambika R} \\ Professor, Department of Electronics and Communication Engineering, \\ BMS Institute of Technology \& Management, Bengaluru, India
}

\begin{abstract}
The conditions within an ecosystem as well as weather of a field affect crop productivity greatly. Different weather conditions produce different effects and impact on the quality of the crop yield or on the ecosystem. Weather elements form a chain reaction, as the atmosphere is not the only one being affected, atmospheric air temperature, vapor pressure and relative humidity or moisture content can act together and form diverse effects on crops. These diverse effects in turn reduces radiation which is necessary for plants, or increases rainfall patterns. Consistent high temperatures can increase the heat transfer to local water bodies in addition to heating the air. Monitoring the climate and the weather conditions are important not only as an environmental baseline, but to maintain quality working conditions, marine studies and recreational safety. The parameters of climate are measurable, for example, atmospheric vapor pressure, temperature, precipitation and solar radiation can be captured and recorded daily on the Arduino Sensor Integrated Drone. Means and extreme datasets, maximum and minimum weather trends with deviations of lengthy time series would be calculated for each of these climate parameters which are considered in this study. These results are a simple form of climate indices, as they already describe changes in climate. All the readings and datasets are recorded on a cloud platform, as well as, in an installed microchip on the drone. Data synchronization is done with Arduino Programming Rule.
\end{abstract}

Key words: Arduino, sensor, weather data, environment.

Cite this Article: Dr. Anil Kumar D and Dr. Ambika R, Drone Integrated Weather Sensors for Agriculture Purpose. International Journal of Electrical Engineering and Technology, 11(5), 2020, pp. 83-90.

http://iaeme.com/Home/issue/IJEET?Volume $=11 \&$ Issue $=5$ 


\section{INTRODUCTION}

Climate and weather data monitoring systems provide vivid and exact information for farming purposes which goes beyond weather stations. Interesting enough, every farmer needs to know and monitor the weather if high productivity has to be met.

Agricultural productivity relies directly and indirectly on the weather and climatic conditions. Therefore, failure to adhere to the weather conditions before, during and after farming renders crop production susceptible to unsuspected disease attacks that are caused or influenced by certain weather conditions. This study is intended to meet the demand for easyto-use, easy-to-access, and accurate weather data for farmers using a drone (UAV) with mounted Arduino Sensors to give accurate climate information to farmers in crop production especially rainfall, solar (duration and intensity) and temperature.

This Arduino drone is being built and developed with state of-the-art multi-parameter weather sensors which focus on four main weather parameters-radiation, temperature, rainfall, and humidity which is an all in one compact lightweight instrument called Arduino Drone. These readings of weather parameters focus on the above four data sets as they have directly or indirectly effect on crop production.

\section{LITRATURE SURVEY}

Literature Survey is an important phase in the system development life cycle as we collect and acquire the necessary information to handle or develop a project during this phase. A literature review is a description of literature relevant to a particular field or topic. It gives an overview of what has been said, who the key writers are, what are the prevailing theories and hypothesis and what methods and what methodologies are appropriate and useful.

In this chapter research is done prior to taking up the project and understanding the various methods that were used previously. A detailed analysis of the existing system was performed. This study helped to identify the benefits and drawbacks of the existing systems. In agriculture usage of drones is very limited. Presently the drones are used in following areas

- Soil and Field Analysis - Drones obtain 3D maps of existing soil which will help farmers to determine the most effective patterns for planting, managing crops, soil and more.

Seed Planting - Drone planting is new technology which helps to minimize the need for onthe-ground planting, which can be costly, time intensive and strenuous work.

- Crop Spraying and Spot Spraying - Drones can be equipped with large reservoirs, which can be filled with fertilizers, herbicides, or pesticides. Using drones for crop spraying is much safer and cost-effective.

- Crop Mapping and Surveying - One of the biggest advantages of using drone technology is the ease and effectiveness of large-scale crop and acreage monitoring. With drone mapping and surveying, technology decisions can now be made based on real-time data, not outdated imagery, or best-practice guesswork.

- Irrigation Monitoring and Management - Drones that are equipped with thermal cameras can help to spot irrigation issues, or areas that are receiving too little or excessive moisture. With this information, crops can be better laid out to maximize drainage, adhere to natural land runoff, and avoid water pooling, which can damage sensitive crops.

This project will give first-hand information about the weather to farmers directly on the farm so their mobile and crop performances can be monitored. 


\section{BLOCK DIAGRAM OF PROPOSED SYSTEM}

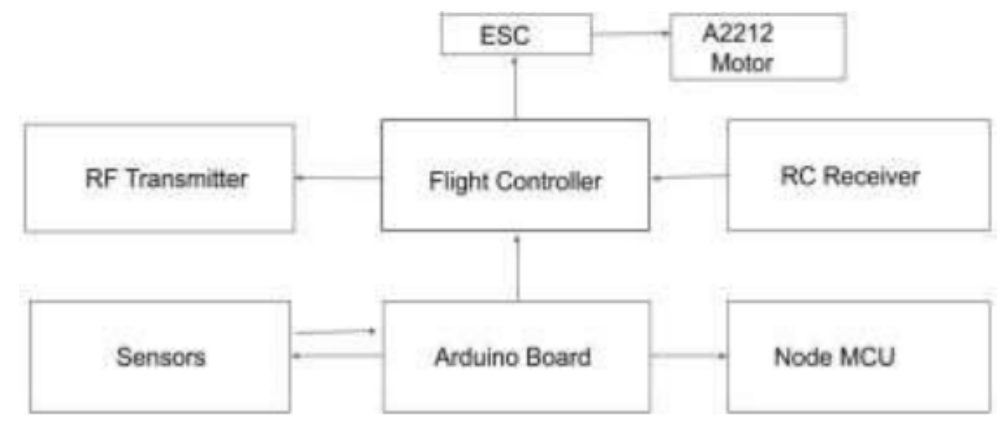

Figure 1 Proposed System

\section{COMPONENTS AND EXPERIMENTAL PROCEDURES}

1. For our experiment, we designed a quadrotor using the propeller frame F450 frame.

2. Flight Controller- CC3D autopilot running Ardupilot firmware as our flight controller.

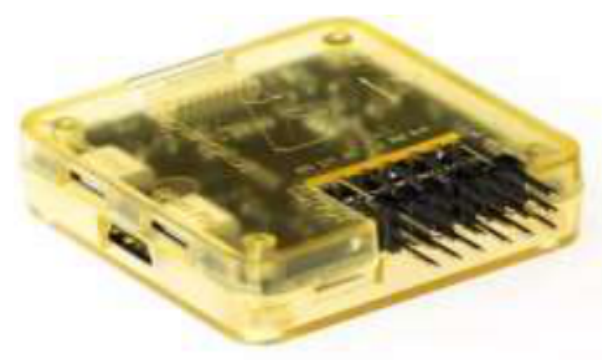

Figure 2 Flight Controller

3. RC Receiver and RF Transmitter- FS-iA6B This receiver system uses low power electronic components and a sensitive receiver chip for the drone. The RF Transmitter modulation uses intermittent signals thus reducing even more power consumption.

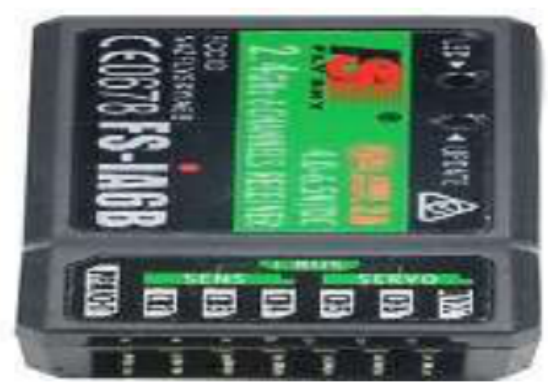

Figure 3 RC Receiver and RF Transmitter

4.Electronic Speed Controller (ESC)- An Electronic Speed Controller (ESC) is an electronic circuit that acts as the interface between the pilot's commands and the individual drone motors.

5. DHT11- is a low-cost humidity and temperature sensor with a single wire digital interface. The sensor is calibrated and doesn't require extra components so you can get right to measuring relative humidity and temperature. 

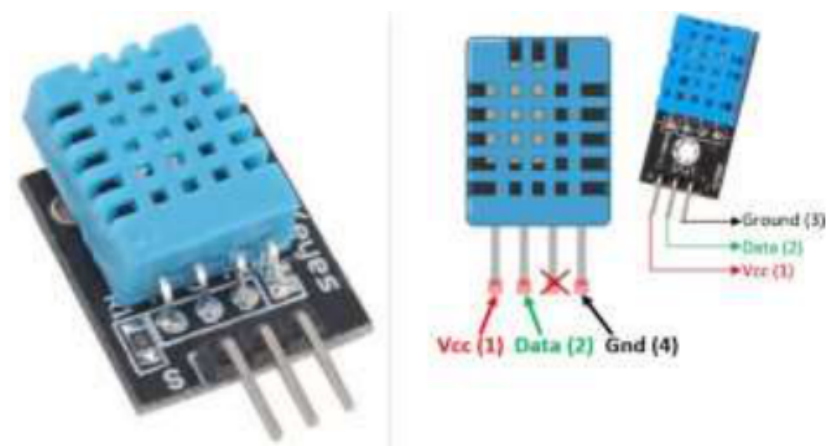

Figure 4 DHT11

6. BMP180 - The BMP180 barometric pressure sensor is a great sensor that can be used to predict the weather, detect altitude, and measure vertical velocity. It is perfect for weather stations, remote controlled vehicles, weather balloons, and lots of other projects. It is an extremely sensitive sensor too. As you will see in a minute, it can detect changes in altitude of just a few inches.

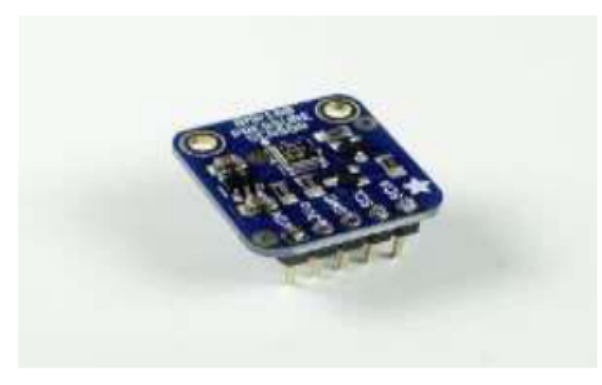

Figure 5 BMP 180

7. Raindrop Sensor- is a tool used for sensing rain. It consists of two modules, a rain board that detects the rain and a control module, which compares the analog value, and converts it to a digital value. The raindrop sensors can be used in the automobile sector to control the windshield wipers automatically, in the agriculture sector to sense rain and it is also used in home automation systems.

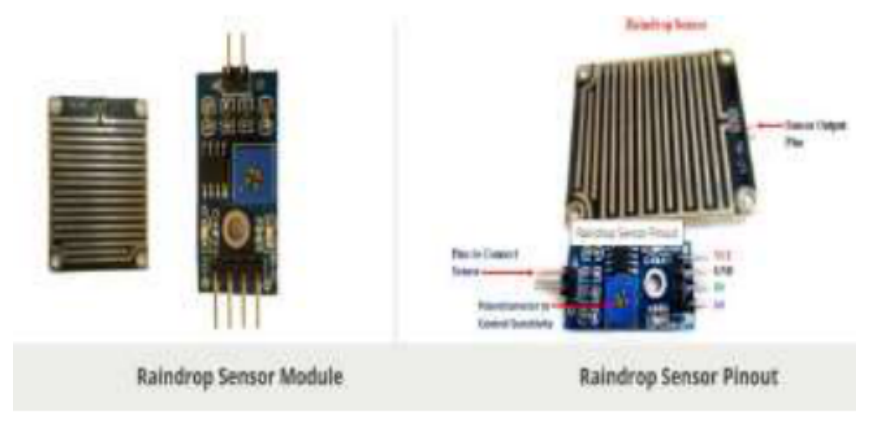

Figure 6 Raindrop Sensor

8. ESP8266(Node MCU) -NodeMCU is an open source IoT platform. It includes firmware which runs on the ESP8266 Wi-Fi SoC from Espressif Systems, and hardware which is based on the ESP-12 module. The term "NodeMCU" by default refers to the firmware rather than the development kits. The firmware uses the Lua scripting language. It is based on the eLua project, and built on the Espressif Non-OS SDK for ESP8266. It uses many open source projects, such as lua-cjson and SPIFFS. 


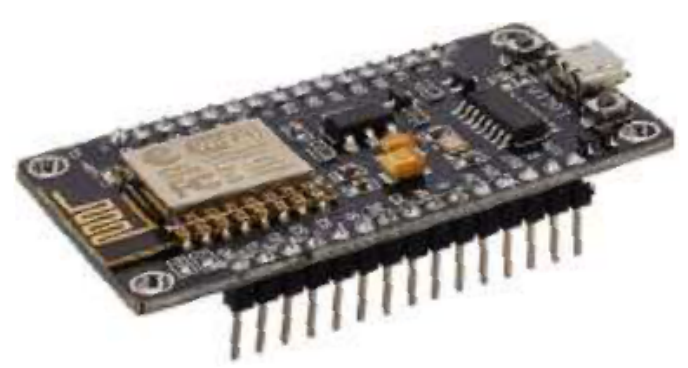

Figure 7 ESP8266(Node MCU)

\section{METHODOLOGY}

Data Captured by the Arduino Board- When the drone flies, the weather data is recorded by the sensors which is mounted on the Arduino board. The temperature and humidity sensor (DHT11) consist of a capacitive humidity sensing element and a thermistor for sensing temperature. The humidity sensing capacitor has two electrodes with a moisture holding substrate as a dielectric between them. Change in the capacitance value occurs with the change in humidity levels. The IC measure, process this changed resistance values and change them into digital form.For measuring temperature this sensor uses a Negative Temperature coefficient thermistor, which causes a decrease in its resistance value with increase in temperature. To get larger resistance value even for the smallest change in temperature, this sensor is usually made up of semiconductor ceramics or polymers. The rain drop sensor has two cases: -

Case1: When the input of the inverting terminal is higher than the input of the non-inverting terminal.

Case2: If the input of the inverting terminal is lower than the input of the non-inverting terminal.

When the rain board module's surface is exposed to rainwater, the surface of the rain board module will be wet, and it offers minimum resistance to the supply voltage. Due to this, the minimum voltage will be appearing at the non-inverting terminal of LM393 Op-Amp. The comparator compares both inverting and non-inverting terminal voltages. If the condition falls under case (1), the output of the Op-Amp will be digital LOW. If the condition falls under case (2), the output of the Op-Amp will be digital HIGH. The below diagram shows the equivalent circuit of both the conditions.

The pressure sensor senses the pressure using (BMP180). The BMP180 is a piezoresistive sensor that detects pressure. Piezoresistive sensors are made up of a semiconducting material (usually silicon) that changes resistance when a mechanical force like atmospheric pressure is applied. The BMP180 measures both pressure and temperature, because temperature changes the density of gasses like air. At higher temperatures, air is not as dense and heavy, so it applies less pressure on the sensor. At lower temperatures, air is denser and weighs more, so it exerts more pressure on the sensor. The sensor uses real-time temperature measurements to compensate for the pressure readings for changes in air density. The BMP180 outputs an uncompensated temperature (UT) value and an uncompensated pressure

(UP) value. The temperature measurement is taken first, followed by a pressure measurement. This flow chart outlines the steps the sensor takes when performing a measurement: 
All the sensors within the Smart Arduino Drone would be calibrated using the Arduino Software and data analysis done by Arduino. After all the sensors are mounted on the Arduino board, the Arduino board needs to be aligned, connected to a data logger (computer) and power source (Battery). Flying height of the drone depends on the individual but it is estimated that flying higher allows you to cover more ground in a shorter amount of time with less battery usage.

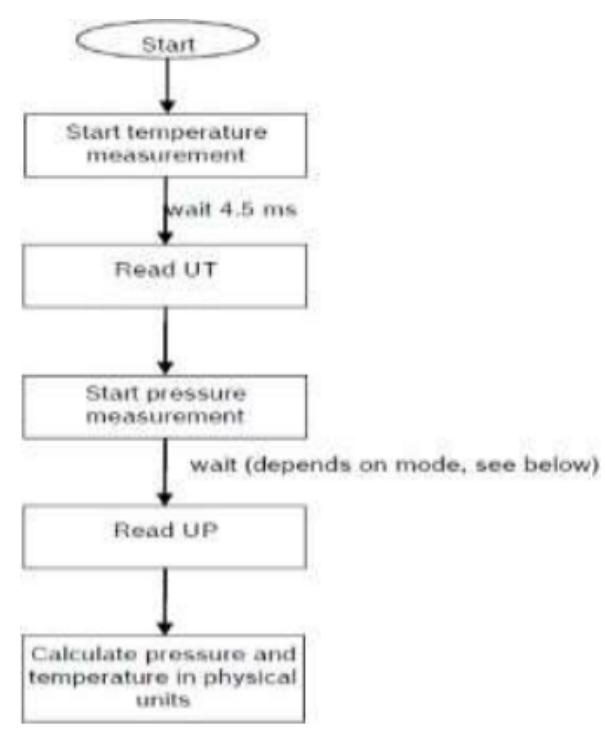

Figure 8 Flowchart for the steps taken by sensor when performing measurement

Data Analysis using Software- The software or any program used for the operation of any system can be written in any language considering various factors. The choice is made on the basis of following decisive factors: -

- Required execution speed

- Accurate control of peripheral devices

- Programming experience of team members

- Time available

Assembly language is considered to be the best for projects that need minimum memory, the highest execution speed, and precise control of peripheral devices but since writing in this language is a tedious task with more knowledge in $\mathrm{C}$ programming, we choose to write our source code in the $C$ language. Not only that $C$ programming is used knowing us the more about it but it has also several advantages over assembly language.

\subsection{Choosing $\mathrm{C}++$ language}

$\mathrm{C}++$ is a powerful, flexible language that provides fast program execution and imposes few constraints on the programmer. It allows low level access to information and commands while still retaining the portability and syntax of a high-level language. These qualities make it a useful language for both system programming and general-purpose programs. Its flexibility comes from the many ways the programmer has to accomplish the same tasks. $\mathrm{C}++$ includes bitwise operators along with powerful pointer manipulation capabilities. $\mathrm{C}++$ imposes few constraints on the programmer. The main area this shows up is in C's lack of type checking. This can be a powerful advantage to an experienced programmer but a dangerous disadvantage to a novice. Another strong point of $\mathrm{C}++$ is its use of modularity. Section of code can be stored in libraries for re-use in future programs. This concept of modularity also 
helps with ' $\mathrm{c}++$ ' portability and execution speed. The core $\mathrm{C}++$ language leaves out many features included in the core of other languages. These functions are instead stored in the $\mathrm{C}++$ standard Library where they can be called on when needed. An example of this concept would be C's lack of built in I/O capabilities. I/O functions tend to slow down program execution and also be machine independent when 23 running optimally. For these reasons, they are stored in a library separately from the $\mathrm{C}$ language and only included when necessary.

- Arduino IDE

- Blynk android application in android mobile phone.

Blynk was designed for the Internet of Things. It can control hardware remotely, it can display sensor data, it can store data, visualize it and do many other cool things.

There are three major components in the platform:

Blynk App - allows you to create amazing interfaces for your projects using various widgets we provide.

Blynk Server - responsible for all the communications between the smartphone and hardware. You can use our Blynk Cloud or run your private Blynk server locally. It's opensource, could easily handle thousands of devices and can even be launched on a Raspberry Pi.

Blynk Libraries - for all the popular hardware platforms - enable communication with the server and process all the incoming and outcoming commands.

\section{RESULTS AND DISCUSSION}

\subsection{Picture of Module}

It is based on the autopilot data generated, the Arduino Sensor Integrated Drone will predict the average means of temperature and humidity, rainfall, wind pressure. This Drone in agriculture will be a big boost to farmers since it carries the potential of completely transforming and revolutionizing the farming and agriculture industry.
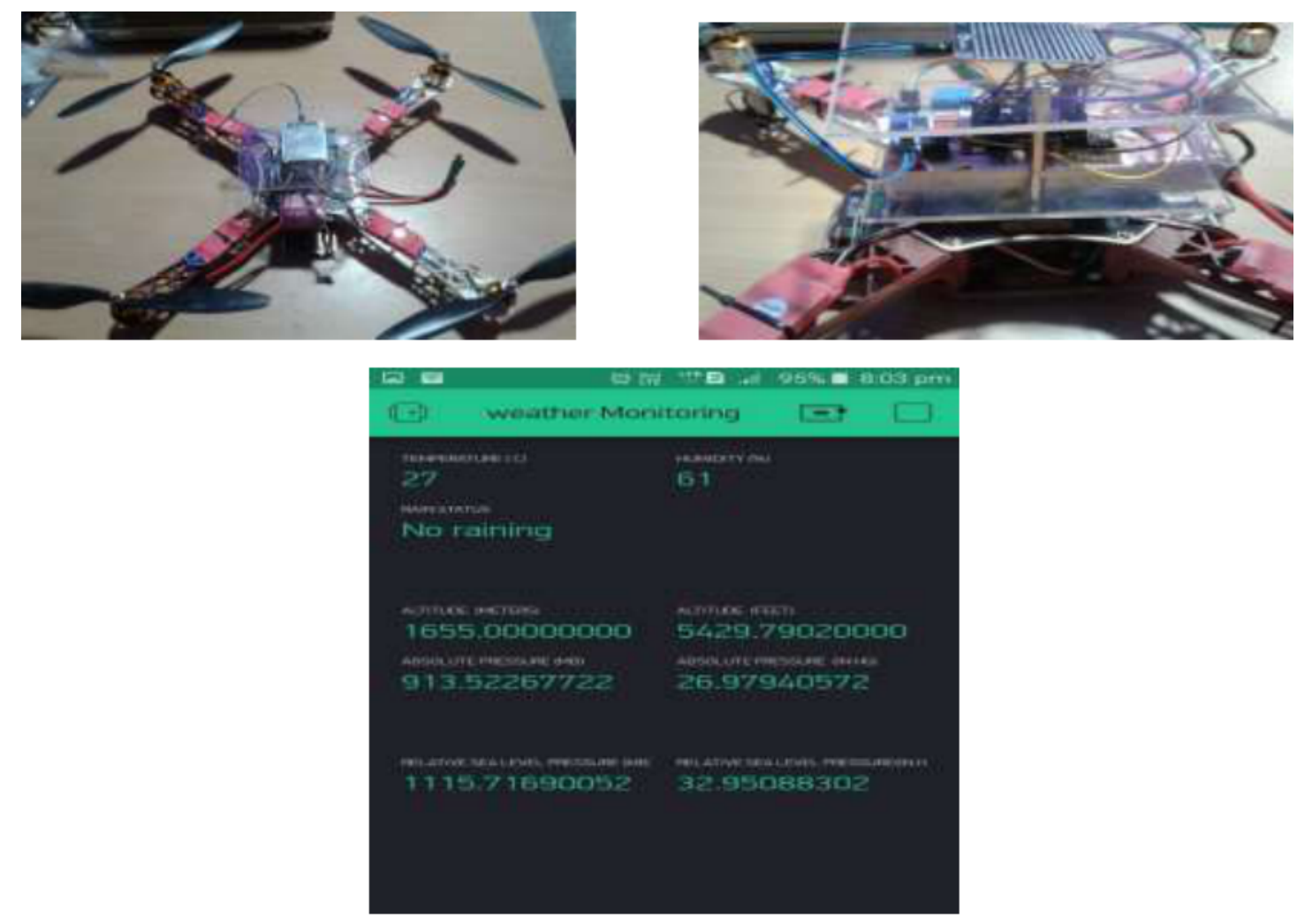

Figure 9 
Weather parameter reading, tracking weather and estimating yields, and then collecting the data and analyzing it for prompt action are some elements considered in this project

\section{CONCLUSIONS}

This Arduino Integrated Drone can mechanize every step of farming and eliminate the costs of human errors and enable farmers to react quickly to threats (such as drought conditions and flood), helping with maximization of income and returns on investment crop productivity.

This Drone in agriculture is a big boost to farmers because it carries the potential of completely transforming and revolutionizing the farming and agriculture industry.

\section{FUTURE ENHANCEMENT}

Weather parameter reading, soil health scans, monitoring crop health, applying fertilizers and watering the fields, even tracking weather and estimating yields, and then collecting the data and analyzing it for prompt action are some elements considered in this ongoing project.

\section{REFERENCES}

[1] Vining KC. (1990). Effects of weather on agricultural crops and livestock: An overview. International Journal of Environmental Studies, 36 (1-2):27-39

[2] Darack E. (2012). UAVs: The new frontier for weather research and prediction. Weatherwise, 65(2): 20-27

[3] Acheampong AA, Fosu C, Amekudzi LK, Kaas E. (2017). Perceptible water comparisons over Ghana using PPP techniques and reanalysis data. South African Journal of Geomatics, $6(3): 449$

[4] Jensen J. (2019). Agricultural Drones: How Drones Are Revolutionizing Agriculture and How to Break into this Booming Market. https:/uavcoach.com/agricultural-drones/

[5] Herrmann JW, Lin ET, Al E. (2001). Affordable space systems manufacturing: Intelligent synthesis. Paper presented at the CD-ROM ASME Design Engineering Technical Conferences.

[6] Arakliotis S, Nikolos DG, Lawris KE. (2016). A Rule Based Arduino Programming System for Young Students. Paper presented at the International Conference on Modern Circuits and Systems Technologies

[7] Curry JM, Ohnson ER, Tarnes JH. (2015). Effect of dropped plies on the strength of graphiteepoxy laminates. AIAA Journal, 30(2):449-456 Cereveny R.Hamilton and the hurricane. Weatherwise. 2016;69(5):42-48

[8] S.Khan, L.Arago and J. Iriarte,"A uavlidar system to map amazonian rainforest and its landscape transformations," International Journal of Remote Sensing, vol. 38, no. 8-10,pp. 2313-2330, 2017.

[9] X. Guo, S. Denman, C. Fookes, L. Meijias, and S. Sridharan,”Automatic uav forced landing site detection using machine learning," in 2014 International conference on digital image computing: Techniques and Applications (DICTA), Nov 2014,pp. 1-7.

[10] Polly, J.; Hegarty-Craver, M.; Rineer, J.; O’Neil, M.; Lapidus, D.; Beach, R.; Temple, D.S. The use of Sentinel1 and -2 data for monitoring maize production in Rwanda. In Proceedings of the Remote Sensing for Agriculture, Ecosystems, and Hydrology XXI. Int. Soc. Opt. Photonics 2019, 11149, 111491.

[11] Zhang, C.; Kovacs, J.M. The application of small unmanned aerial systems for precision agriculture: A review. Precis. Agric 2012, 13, 693-712.

[12] Hung, C.; Xu, Z.; Sukkarieh, S. Feature Learning Based Approach for Weed Classification Using High Resolution Aerial Images from a Digital Camera Mounted on a UAV. Remote Sens. 2014, 6, 12037-12054. 